\title{
Publisher's Note: Optomechanics for absolute rotation detection [Phys. Rev. A 94, 013808 (2016)]
}

\author{
Sankar Davuluri \\ (Received 11 August 2016; published 22 August 2016)
}

DOI: 10.1103/PhysRevA.94.029906

This paper was published online on 5 July 2016 with errors in Eqs. (28) and (29). Equation (28) should read as

$$
\Omega_{\mathrm{min}}^{2}=\frac{\zeta l \omega_{m}^{2}}{4 \omega_{o} r_{o} \sqrt{I_{\mathrm{opt}} t_{m}}}\left\{3+2 \frac{\gamma_{m}}{\omega_{m}}\left(1+\frac{2}{e^{\hbar \omega_{m} / k T}-1}\right)\right\}^{1 / 2} \approx \frac{\zeta l \omega_{m}^{2}}{4 \omega_{o} r_{o} \sqrt{I_{\mathrm{opt}} t_{m}}} \sqrt{3+4 \frac{\gamma_{m}}{\omega_{m}} \frac{k T}{\hbar \omega_{m}}} .
$$

Equation (29) should read as

$$
\Omega_{\min }^{2}=\frac{1}{r_{o} \sqrt{m t_{m}}} \sqrt{2 \gamma_{m} k T}
$$

The equations have been corrected as of 12 August 2016. The equations are incorrect in the printed version of the journal. 